\title{
Les groupes sanguins des poneys kirdi (Tchad)
}

\author{
par $M^{\mathrm{me}}$ L. PODLIACHOUK ef R. QUEVAL
}

La population chevaline de la République du Tchad est rattachée à deux grandes races originelies : aryenne et mongolique à l'exception des poneys Kirdi - race autochtone - dont l'aire de répartition géographique est limitée : au Sénégal (Baol), au Dahomey (cheval Cotocoli), aux falaises de Bandiagara et au Tchad où la zone d'élevage de ces poneys se situe de part et d'autre du fleuve Logone, entre $8,5^{\circ}$ et $10^{\circ}$ de latitude Nord.

Ce poney est appelé cheval Kirdi, parce qu'il est élevé par les populations fétichistes («Kirdi » en arabe local), ou plus rarement cheval Sara ou cheval Mbaye.

Dans la Shanga et le Cameroun, il est connu comme cheval Lakka («Lakka » est dit en langue Peul pour désigner certaines tribus fétichistes).

Le poney Kirdi est trapu, massif, de très petite taille $(1,10 \mathrm{~m}$ à $1,25 \mathrm{~m})$; sub-longiligne, ellipométrique, d'un poids moyen de 150 à 200 kilogrammes.

La tête est massive et lourde, longue, peu chargée de ganaches. Le front est large et plat. L'œil est couvert, expressif; les arcades orbitaires ne sont pas très saillantes.

L'encolure est courte, épaisse, la poitrine est peu étriquée : les épaules sont plaquées; le dos droit et large; le rein court. La croupe est large, carrée et légèrement tranchante. La queve est longue.

La robe est variable mais le plus souvent foncée sans taches blanches, parfois grise ou même pie (rare).

Ces chevaux sont extrêmement rustiques, solides et résistants $(1,2)$.

Reçu pour publication : octobre 1961.

Rev. Elev. Méd. vét Pays trop., 1961, 14, no 4.

\author{
MATÉRIEL ET TECHNIQUE \\ I) Globules rouges
}

Les animaux furent saignés avec les précautions habituelles d'aseptie par voie intraveineuse. Les échantillons de sang furent récoltés sur citrate de soude à 10 p. 100 et conservés à $+4^{\circ} \mathrm{C}$.

\section{2) Sérums}

Le sang total récolté dans des flacons stériles est laissé 24 heures à la température du laboratoire pour permettre l'exsudation du sérum à partir du caillot. Ce sérum est ensuite centrifugé, décanté et conservé à $20^{\circ} \mathrm{C}$.

\section{3) Sérums de référence}

Les sérums de référence ont été isolés par l'un de nous à l'Institut Pasteur de Paris et conservés congelés à $-20^{\circ} \mathrm{C}$.

\section{4) La réaction d'agglutination}

Dans des tubes à hémolyse les sérums sont mis en présence de globules rouges en susperision à 1 p. 100 (lavés 2 fois dans de l'eau physiologique) sous un volume de $0,05 \mathrm{ml}$ de sérum pour $0,05 \mathrm{ml}$ de suspension globulaire.

La première lecture est effectuée après $30 \mathrm{mn}$ de contact à la température du laboratoire, à l'œil nu, à l'aide d'un miroir concave.

La deuxième lecture est effectuée après $1 \mathrm{mn}$ de centrifugation à 1.500 tours.

La deuxième lecture permet, en général, de confirmer ou infirmer les résultats difficiles à interpréter.

\section{RÉSULTATS}

l.'éfude de la répartition des antigènes érythrocytaires chez les chevaux suivant la race a 
TABLEAU 1 - Répartition des antigènes érythrocytaires.

\begin{tabular}{|c|c|c|c|c|c|c|}
\hline \multirow{2}{*}{$\begin{array}{l}\text { Anticènes } \\
\text { érythrocytaires }\end{array}$} & \multicolumn{2}{|c|}{ Race ardennaise } & \multicolumn{2}{|c|}{ Pur-sang anglais } & \multicolumn{2}{|c|}{ Poney Kirdi } \\
\hline & $\begin{array}{c}\text { Nombre } \\
d^{\prime} \text { animaux }\end{array}$ & Fréquence & $\begin{array}{c}\text { Nombre } \\
\text { d'animaux }\end{array}$ & Fréquence & $\begin{array}{c}\text { Nombre } \\
d^{\dagger} \text { animaux }\end{array}$ & Fréquence \\
\hline A & 47 & 0,532 & 162 & 0,790 & 40 & 0,625 \\
\hline c & $"$ & 0,723 & $"$ & 0,938 & $"$ & 1,000 \\
\hline D & $"$ & $\underline{0.511}$ & $"$ & 0 & $"$ & 0 \\
\hline $\mathrm{E}$ & $"$ & 0,021 & $"$ & 0,204 & $"$ & 0,225 \\
\hline$F$ & $"$ & 0,638 & $"$ & 0,790 & $"$ & 0,625 \\
\hline H & - & - & $"$ & 0 & $"$ & 0 \\
\hline$J$ & - & - & 62 & $0, \infty 01$ & $"$ & $\underline{0.475}$ \\
\hline L & - & - & - & - & $"$ & 0 \\
\hline
\end{tabular}

été abordée antérieurement par plusieurs auteurs qui ont observé que la fréquence des divers antigènes globulaires varie sensiblement avec la population étudiée. Cependant, comme chacun des auteurs utilisaient une nomenclature qui lui était propre, il n'est pas possible de comparer les résultats obtenus entre eux (3).

Dans une étude précédente nous avons déterminé le groupe sanguin de 59 chevaux du Tchad appartenant à une population hétérogène $(5,6)$.

L'étude comparative de la répartition des antigènes chez ces chevaux avec ceux de l'annexe de l'Institut Pasteur de Garches (4) - lesquels constituent également une population hétérogène - ne présente aucune différence significative.

Duns le présent travail nous avons examiné 40 poneys Kirdi lesquels constituent une population homogène. Les groupes sanguins de ces animaux ont été déterminés à l'aide des 8 anticorps de référence anti $A, C, D, E, F, H$, J et $L$.

Les résultats sont rapportés dans le tableau I ef comparés à ceux obtenus par l'un de nous chez les chevaux de race ardennaise et les pursang. Notons que le nombre d'antigènes pour lesquels les fréquences ont été établies dans diverses populations varie avec l'époque à laquelle l'étude a eu lieu. Ainsi la race ardennaise a été examinée quand les antigènes $H, J$ et $L$ n'étaient pas encore découverts.

Des résultats obtenus, il ressort que la race ardennaise est caractérisée par la fréquence très élevée du facteur $D$ qui est absent chez le pur-sang et le poney Kirdi. Ce dernier est caractérisé par une fréquence très élevée de l'antigène $J$ qui est de 0,475 tandis que chez les pur-sang elle n'est quc de 0,081 .

La fréquence du facteur $C$ et $E$ chez les poneys Kirdi est plus élevée que chez les pur-sang et la race ardennaise. Les facteurs $H$ et $D$ sont complètement absents comme chez les pur-sang.

[n outre nous avons recherché les isoagglutinines naturelles dans le sérum de ces poneys. Parmi les 15 animaux $A$ négatifs, le sérum de 10 contient une isoagglutinine naturelle anti-A de faible titre (ne dépassant pas le 1/4).

Comme nous l'avons observé dans des travaux antérieurs l'isoagglutinine anti-A est souvent présente dans le sérum d'animaux $A$ négatifs.

Cette règle n'est pas valable pour d'autres facteurs tels que $D, E, H$, J et $L$. Les isoagglulinines correspondantes ont été trouvées une ou deux fois au cours de l'examen d'environ 1.000 animaux

Cette étude nous permet de conclure que la population homogène des poneys Kirdi est caractérisée par une fréquence très élevée du facteur érythrocytaire J et par l'absence des facteurs $\mathrm{D}$ et $\mathrm{H}$.

\section{Contrc d'études des groupes sanguins} des animaux, Laboratoire d'hématologie et des groupes sanguins, Institut Posteur (Paris) et

Institut d'élevage et de médecine vétérinaire des pays tropicaux : Laboratoire de recherches vétérinaires de Farcha, Fort-Lamy (Tchad). 


\section{RÉSUMÉ}

L'étude des groupes sanguins de 40 chevaux de race poney Kirdi a permis de constater la fréquence très élevée du facteur érythrocytaire $\mathrm{J}(0,475)$ et l'absence de facteurs $\mathrm{D}$ ef $\mathrm{H}$.

\section{SUMMARY}

The bload groups of the Kirdi breed of pony

A study was made of the blood groupings of 40 individuals of a breed of Tchad horses, known as Kirdi, from which it was shown that there was a high prevalence of the erythrocyte factor J. (0.475), and the absence of factor $D$. and $H$.

\section{RESUMEN}

\section{Los grupos sanguineos de los poneys kirdi (Tchad)}

El estudio de los grupos sanguineos de 40 caballos de raza poney Kirdi ha permitido de constatár una frecuencia muy elevada del factor eritrocitario $J(0,475)$ y la ausencia de factóres $D$ y $H$.

\section{BIBLIOGRAPHIE}

1. DOUTRESSOULLE (G.). - Bull. Serv. zootech. Epiz. A. O. F., 1941, 4 (3 et 4) : 145.

2. PECAUD (J.). - Monogr. Serv. Elev. Tchad, 1926 (non publiée).

3. PODLIACHOUK (L.). - Thèse de Doctorat ès-Sciences, Paris, 1957.
4. PODLIACHOUK (L.). - Ann. Inst. Pasteur, 1958, $95: 7-22$.

5. PODLIACHOUK (L.) et QUEVAL (R.). Ann. Inst. Posteur, 1961, $100: 133-6$.

6. PODLIACHOUK (L.) et QUEVAL (R.). - - Rev Elev. Méd. vét. Pays trop., 1961, 14 : 53-6. 